\title{
Laparoscopic removal of retroperitoneal tumor with maneuver of hanging inferior vena cava
}

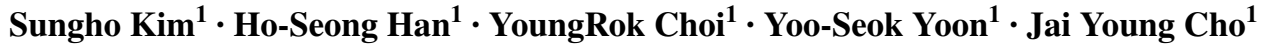

Received: 8 April 2017 / Accepted: 23 October 2017 / Published online: 7 December 2017

(C) Springer Science+Business Media, LLC 2017

\begin{abstract}
Background Laparoscopic resection of retroperitoneal mass is challenging because of its location close to major vessels and a limited range of laparoscopic instruments [1-3]. We report a case of a huge retroperitoneal paraganglioma that was successfully excised laparoscopically using maneuver of hanging IVC

Method A 67-year-old female had abdominal mass detected during routine check-up. She had no symptoms associated mass. Hematologic, biochemical investigations, and hormone tests reveal normal results. Preoperative CT shows retroperitoneal tumor, measuring $6.1 \mathrm{~cm}$ in diameter, which was closely adhered to right adrenal gland and seemed to originate from adrenal gland. Three 12-mm trocars and two 5-mm trocars were used. Laparoscopic views showed that severe adhesion in peritoneal cavity due to previous subtotal gastrectomy for gastric polyp about 30 years ago. After adhesiolysis, the mass was visualized behind inferior vena cava. The mass was carefully dissected and separated from duodenum and inferior vena cava as well as right adrenal gland. Inferior vena cava was retracted with hanging maneuver of IVC with vascular tape. The fibrotic tissues covering the mass were dissected with ligasure ${ }^{\mathrm{TM}}$. The feeding vessels supplying mass were identified and clipped, then subsequently sealed and divided. After complete resection
\end{abstract}

Electronic supplementary material The online version of this article (https://doi.org/10.1007/s00464-017-5969-z) contains supplementary material, which is available to authorized users.

Ho-Seong Han

hanhs@snubh.org

1 Department of Surgery, Seoul National University Bundang Hospital, Seoul National University College of Medicine, 166 Gumi-ro, Bundang-gu, Seongnam, Gyeonggi 463-707, South Korea of mass, the specimen was inserted into plastic retrieval bag and extracted via extended umbilical port. And then Jackson Pratt drain was placed around inferior vena cava.

Result There was no intraoperative transfusion and complications during laparoscopic retroperitoneal excision for paraganglioma. The operation time was $190 \mathrm{~min}$, and estimated blood loss was $100 \mathrm{ml}$. The patient was discharged on postoperative 5th day without complications. Final pathologic result was paraganglioma of $7.5 \mathrm{~cm}$ sized with PASS score 4 (pheochromocytoma of the adrenal gland scaled score).

Conclusion Laparoscopic surgery for huge retroperitoneal paraganglioma behind IVC was successfully performed with the maneuver of hanging IVC. This procedure could be useful with appropriate laparoscopic technique and proper patient selection.

Keywords Laparoscopic $\cdot$ Retroperitoneal $\cdot$ Inferior vena cava

Compliance with ethical standards

Disclosures Authors Sungho Kim, Ho-Seong Han, YoungRok Choi, Yoo-Seok Yoon, Jai Young Cho, have no conflict of interest or financial ties to disclose.

\section{References}

1. Nozaki T, Iida H, Morii A, Fujiuchi Y, Okumura A, Fuse H (2013) Laparoscopic resection of adrenal and extra-adrenal pheochromocytoma. J Endourol Endourol Soc 27(7):862-868

2. Ploussard G, Xylinas E, Paul A, Gillion N, Salomon L, Allory Y et al (2009) Is robot assistance affecting operating room time compared with pure retroperitoneal laparoscopic radical prostatectomy? J Endourol Endourol Soc 23(6):939-943

3. Xu W, Li H, Ji Z, Yan W, Zhang Y, Zhang X et al (2016) Retroperitoneal laparoscopic management of paraganglioma: a single institute experience. PLoS ONE 11(2):e0149433 